\title{
CURVES AND VECTOR BUNDLES ON QUARTIC THREEFOLDS
}

\author{
Enrique Arrondo and Carlo G. Madonna
}

\begin{abstract}
In this paper we study arithmetically Cohen-Macaulay (ACM for short) vector bundles $\mathcal{E}$ of rank $k \geq 3$ on hypersurfaces $X_{r} \subset \mathbb{P}^{4}$ of degree $r \geq 1$. We consider here mainly the case of degree $r=4$, which is the first unknown case in literature. Under some natural conditions for the bundle $\mathcal{E}$ we derive a list of possible Chern classes $\left(c_{1}, c_{2}, c_{3}\right)$ which may arise in the cases of rank $k=3$ and $k=4$, when $r=4$ and we give several examples.
\end{abstract}

\section{Introduction}

Let $X=X_{r} \subset \mathbb{P}^{4}$ be a smooth hypersurface of degree $r \geq 1$ and let $H$ be the class of a hyperplane section. It is well known that

$$
\operatorname{Pic}\left(X_{r}\right) \cong \mathbb{Z}[H] \cong \mathbb{Z}
$$

and hence we may identify any line bundle $\mathcal{L}$ on $X_{r}$ with the sheaf $\mathcal{O}_{X_{r}}(n H) \cong$ $\mathcal{O}_{X_{r}}(n)$ for some $n \in \mathbb{Z}$. We recall the following:

Definition 1.1. Let $\mathcal{E}$ be a rank $k$ vector bundle on $X_{r}, r \geq 1$. We call $\mathcal{E}$ arithmetically Cohen-Macaulay (ACM for short) if $h^{i}\left(X_{r}, \mathcal{E}(n)\right)=0$ for all $i=1,2$ and $n \in \mathbb{Z}$, where $\mathcal{E}(n):=\mathcal{E} \otimes \mathcal{O}_{X_{r}}(n)$, under the identification of (1.1).

In a previous work [14] the second author showed that a rank two ACM vector bundle on $X_{r}$ splits as a direct sum of two line bundles with only few possible exceptions. Specifically, for each $r$, all the possible first Chern classes of such an undecomposable bundle (up to a twist with a line bundle) were given.

For low values of $r$, i.e., $r=1,2,3,4$, a complete classification of undecomposable rank two ACM vector bundles is known. We refer the interested reader to [4], [11], [15], [22] for more details on these cases. When $r=5$ the possible

Received September 12, 2007; Revised January 11, 2008.

2000 Mathematics Subject Classification. 14J60.

Key words and phrases. quartic threefold, ACM bundle, projectively normal curve.

The first author was supported in part by the project BFM2003-03971/MATE funded by the Spanish MCYT. The second author was supported in part by the projects I3P-CSIC and MTM2007-67623 founded by the Spanish MEC. 
Chern classes of such an undecomposable bundle where found in [16] while, when $r \geq 6$ and $X_{r}$ is general, the results of [7] (for the case $r=6$ ) and of [13] (for any $r \geq 6$ ) ensure the non existence of such bundles. Similar results were obtained for more general threefolds and in particular a complete classification of rank two ACM vector bundles on prime Fano threefolds of index 1 was given in [10] and [5]. Also in some cases the corresponding moduli spaces are described (see [12] for a comprehensive account of the known results).

On the other hand, for the higher rank case very little is known even in the cases of low degree hypersurfaces. When the rank is bigger than or equal to 3 , it is known that there are no undecomposable ACM vector bundles if $r=1,2$, while as far as we know, only the cubic case was considered in [4] under the following natural assumption (which we generalize to arbitrary $r$ ):

Definition 1.2. We say that a rank $k \geq 2$ vector bundle on $X_{r} \subset \mathbb{P}^{4}$ satisfies condition $\star$ if the following conditions hold:

(i) $h^{0} \mathcal{E}(-1)=0$ and $h^{0} \mathcal{E} \geq k$;

(ii) there exist $k-1$ global sections of $\mathcal{E}$ whose dependency locus has codimension equal to two;

(iii) $\mathcal{E}$ has not trivial summands.

In this paper we will consider mainly the cases of low rank, (precisely $k=$ $3,4)$ when $r=4, X=X_{4}$ is general, and the above condition $\star$ is satisfied. Notice, it is easy to produce examples of higher rank $(>3) \mathrm{ACM}$ vector bundles by using extension classes. Specifically, starting with a pair $\left(\mathcal{E}^{\prime}, \mathcal{E}^{\prime \prime}\right)$ of rank $k$ and $k^{\prime} \mathrm{ACM}$ bundles on $X_{r}$, one may always consider extensions

$$
0 \rightarrow \mathcal{E}^{\prime} \rightarrow \mathcal{E} \rightarrow \mathcal{E}^{\prime \prime} \rightarrow 0
$$

to get a rank $k^{\prime}+k^{\prime \prime} \mathrm{ACM}$ bundle on $X_{r}$. Of course, since line bundles on $X_{r}$ are ACM, to get such non trivial extension classes it is necessary to have $k, k^{\prime} \geq 2$. We will give several examples of rank four ACM bundles satisfying condition $\star$ when $r=4$ obtained in this way in Section 3 .

Since such a construction was already used for the case $r=3$ in [4] and for the case $r=5$ in [17], one could state the following:

Question 1.3. Let $\mathcal{E}$ be a rank four ACM vector bundle satisfying condition * on a general hypersurface $X_{r} \subset \mathbb{P}^{4}$. Is $\mathcal{E}$ necessarily obtained as an extension class of a pair of rank two ACM bundles on $X_{r}$, as in (1.2)?

We give here a negative answer to the above question when $r=4$ and $X_{4}$ is general (see Proposition 4.4). We notice that Question 1.3 still remains open when $r=3$. Moreover when $r=6$ we have not examples at all of rank 4 ACM bundles, while when $r=5$ we know actually only one example, given in [17], which satisfies condition $\star$.

Turning to the case of rank $k=3$, we want to answer the following question:

Question 1.4. Are there rank three ACM bundles satisfying condition $\star$ on a general hypersurface $X_{r} \subset \mathbb{P}^{4}$ of degree $r \geq 4$ ? 
Since in [4] an affirmative answer (see Proposition 4.9) was already given for the case $r=3$ we consider the case $r=4$ here. We give an affirmative answer to this last question when $r=4$. We notice that Question 1.4 remains open when $r \geq 5$. To answer the previous questions, we derive the possible first Chern class of an ACM bundle of rank $k \geq 3$ satisfying condition $\star$ on any smooth hypersurface of degree $r \geq 3$. Then we consider in detail the cases of low rank, i.e., $k=3$ and $k=4$, when $r=4$, and we go on by a case by case analysis. In this direction our main result is the following:

Theorem 1.5. Let $\mathcal{E}$ be an ACM vector bundle of rank $k \in\{3,4\}$ on a general quartic threefold $X_{4} \subset \mathbb{P}^{4}$ which satisfies condition $\star$. Then the possible Chern classes $c_{i}=c_{i}(\mathcal{E})$ and the genus $g=g(C)$ of the curve $C$, dependency locus of $k-1$ global section of $\mathcal{E}$, are given in the following table:

\begin{tabular}{ccccc}
$k$ & $c_{1}$ & $c_{2} \in$ & $c_{3}$ & $g$ \\
\hline 3 & 1 & 5 & 2 & 2 \\
3 & 2 & {$[8,11]$} & $c_{2}-6$ & $c_{2}-2$ \\
3 & 3 & {$[17,18]$} & $2 c_{2}-26$ & $2 c_{2}-12$ \\
3 & 4 & {$[27,28]$} & $3 c_{2}-66$ & $3 c_{2}-32$ \\
4 & 1 & 6 & 4 & 3 \\
4 & 2 & {$[8,12]$} & $c_{2}-4$ & $c_{2}-1$ \\
4 & 3 & {$[16,22]$} & $2 c_{2}-24$ & $2 c_{2}-11$ \\
4 & 4 & {$[28,32]$} & $3 c_{2}-64$ & $3 c_{2}-31$ \\
4 & 5 & {$[44,46]$} & $4 c_{2}-132$ & $4 c_{2}-65$ \\
4 & 6 & 64 & 84 & 203
\end{tabular}

Moreover there are examples of bundles with invariants $\left(k ; c_{1}, c_{2}, c_{3}\right)$ for any of the following quadruples:

$$
\begin{aligned}
& (4 ; 1,6,4),(4 ; 2, \alpha, \alpha-4),(4 ; 3, \beta, 2 \beta-24),(4 ; 4, \gamma, 3 \gamma-64), \\
& (4 ; 5,46,52),(4 ; 6,64,84),(3 ; 1,5,2),
\end{aligned}
$$

where $\alpha \in\{10,11,12\}, \beta \in\{19,20\}$, and $\gamma \in\{29,30,32\}$.

In the case of the rank four, almost all the examples are given by considering extension classes of rank two ACM bundles. It is not so for the case $(4 ; 1,6,4)$, which is constructed by means of the Hartshorne-Serre correspondence (see Proposition 2.1) starting from a suitable smooth projectively normal curve in $X_{4}$ and it is not given by any extension class of any two rank two ACM bundles on $X_{4}$. The example for the rank three case is given in similar way, by showing the existence of the corresponding curve with prescribed invariants and then applying the above mentioned Hartshorne-Serre correspondence.

The paper is organized as follows. In Section 2 we recall some known facts and generalities that we need in the paper. In Section 3 (see Lemma 3.4), we find conditions such that the direct sum (or more generally any extension class) of ACM vector bundles satisfying condition $\star$ is still an ACM vector bundle satisfying condition $\star$, and produce from this several examples of rank four 
bundles on $X_{4}$ (precisely all but one in the list of Theorem 1.5). In Section 4 we use the Hartshorne-Serre correspondence (see Proposition 2.1) between curves and vector bundles to give a characterization (see Proposition 4.1) of when the vector bundle obtained from a curve is ACM and satisfies condition $\star$; using this, we construct the example of rank three and the remaining one of rank four of ACM vector bundles satisfying condition $\star$ on $X_{4}$ of the list of Theorem 1.5. Finally, in Section 5 we give restrictions for the Chern classes of ACM vector bundles on $X_{r}$ satisfying condition $\star$, which when particularized for $r=4$ and rank $k=3,4$ yields the list given in Theorem 1.5 of all possible Chern classes in these ranks.

The preliminary draft of this paper was completed during the second author stay in the Departamento de Álgebra of University Complutense in Madrid, supported by a grant "Mensilità per l'estero" from the "Istituto Nazionale di Alta Matematica F. Severi" (10/2005-03/2006).

\section{Preliminaries and basic facts}

In this paper we work over the field of complex numbers $\mathbb{C}$. Let us consider a smooth hypersurface $X_{r} \subset \mathbb{P}^{4}$ of degree $r \geq 1$ in the complex 4-dimensional projective space $\mathbb{P}^{4}$. Then, as recalled in the introduction $\operatorname{Pic}\left(X_{r}\right) \cong \mathbb{Z}[H]$ where $H$ is the class of a hyperplane section, and the canonical divisor of $X_{r}$ is $K_{X_{r}}=(r-5) H$.

If $P$ denotes the class of a point and $L$ the class of a line, then the intersection products on $X_{r}$ are given by $H^{3}=r P=r, H L=1 P=1, H^{2}=r L$. Let $\mathcal{E}$ be a rank $k$ vector bundle on $X_{r}$. We identify the first Chern class $c_{1}(\mathcal{E})$ of $\mathcal{E}$ with the integer number $c_{1}$ which corresponds to $c_{1}(\mathcal{E})$ under the above isomorphism, i.e., $c_{1}(\varepsilon)=c_{1} H=c_{1}$. In a similar way we identify $c_{2}(\varepsilon)=\operatorname{deg} c_{2}(\varepsilon)=c_{2}$ and $c_{3}(\mathcal{E})=c_{3} P=c_{3}$. Under this identification to any rank $k$ vector bundle $\mathcal{E}$ on $X_{r}$ corresponds a quadruple $\left(k ; c_{1}, c_{2}, c_{3}\right) \in \mathbb{N} \times \mathbb{Z}^{3}$. In this language, our main result gives all the possible quadruples of a rank three and four ACM vector bundle on a smooth quartic threefold which could arise under the condition $\star$ of Definition 1.2.

For further computations we write down explicitly the Chern classes of the bundle $\mathcal{E}(n)=\mathcal{E} \otimes \mathcal{O}_{X_{r}}(n)$ in the following equations:

$$
\begin{aligned}
c_{1}(\mathcal{E}(n)) & =c_{1}+k n, \\
c_{2}(\mathcal{E}(n)) & =c_{2}+r(k-1) n c_{1}+r\left(\begin{array}{l}
k \\
2
\end{array}\right) n^{2}=c_{2}+r n(k-1)\left(c_{1}+\frac{1}{2} n k\right), \\
c_{3}(\mathcal{E}(n)) & =c_{3}+(k-2) n c_{2}+r\left(\begin{array}{c}
k-1 \\
2
\end{array}\right) n^{2} c_{1}+r\left(\begin{array}{c}
k \\
3
\end{array}\right) n^{3} \\
& =c_{3}+(k-2) n\left(c_{2}+\frac{1}{2}(k-1) n r c_{1}+\frac{1}{6} r n^{2} k(k-1)\right) .
\end{aligned}
$$

In the sequel, to perform some computations, we will frequently use the following version of Riemann-Roch theorem for vector bundles: 
Hirzebruch-Riemann-Roch Theorem. If $\mathcal{E}$ is a rank $k$ vector bundle on a smooth hypersurface $X_{r} \subset \mathbb{P}^{4}$ of degree $r \geq 1$ with Chern classes $c_{i}(\mathcal{E})=c_{i} \in \mathbb{Z}$ for $i=1,2,3$, then

$$
\begin{aligned}
\chi(\mathcal{E})= & \frac{1}{6} r c_{1}^{3}-\frac{1}{2} c_{1} c_{2}+\frac{1}{2} c_{3}+\frac{1}{4} r c_{1}^{2}(5-r)-\frac{1}{2} c_{2}(5-r) \\
& +\frac{1}{12}\left((r-5)^{2}+\left(10-5 r+r^{2}\right)\right) c_{1} r+\frac{1}{24} r k(5-r)\left(10-5 r+r^{2}\right) .
\end{aligned}
$$

In particular

$$
\begin{aligned}
\chi\left(\mathcal{O}_{X_{r}}(a)\right)= & \frac{1}{6} a^{3} r+\frac{1}{4} a^{2} r(5-r)+\frac{1}{12} \operatorname{ar}\left((r-5)^{2}\right. \\
& \left.+\left(10-5 r+r^{2}\right)\right)+\frac{r}{24}(5-r)\left(10-5 r+r^{2}\right) .
\end{aligned}
$$

Recall that a vector bundle $\mathcal{E}$ is "decomposable" if there exist $\mathcal{E}^{\prime}$ and $\mathcal{E}^{\prime \prime}$ such that $\mathcal{E} \cong \mathcal{E}^{\prime} \oplus \mathcal{E}^{\prime \prime}$. Otherwise $\mathcal{E}$ is "undecomposable". By the Serre's duality, $\mathcal{E}$ is $\mathrm{ACM}$ if and only if

$$
h^{1} \mathcal{E}(n)=h^{1} \mathcal{E}^{\vee}(-n+r-5)=0 \quad \forall n \in \mathbb{Z}
$$

which is equivalent to the condition $h^{1} \mathcal{E}(n)=h^{1} \mathcal{E}^{\vee}(n)=0 \quad \forall n \in \mathbb{Z}$.

From now on we will consider mainly vector bundles $\mathcal{E}$ of rank $k \geq 3$ for which the condition $\star$ (see Definition 1.2) is satisfied. If $h^{0} \mathcal{E}(-1)=0$ and $h^{0} \mathcal{E}>0$ we will say that $\mathcal{E}$ is "normalized". It is always possible to assume this condition since we may replace $\mathcal{E}$ with $\mathcal{E}(-b)$, where $b=b(\mathcal{E})$ is defined as

$$
b(\mathcal{E})=b=\max \left\{n \in \mathbb{Z}: h^{0} \mathcal{E}(-n)=0\right\} .
$$

We recall also the notion of stability that we will use in the sequel. A rank $k$ vector bundle $\mathcal{E}$ on $X_{r}$ is "stable" (resp. "semistable") if for any subbundle $\mathcal{F} \subset \mathcal{E}$ of rank $m<k$ we have $c_{1}(\mathcal{F}) / m<c_{1}(\mathcal{E}) / k$ (resp. $\leq$ ). In particular if $b(\mathcal{E})=b$ is defined as in $(2.3)$ we have $k b(\mathcal{E})-c_{1}(\mathcal{E})<0$ if $\mathcal{E}$ is stable (resp. $\leq$ ). Indeed, any global section of $H^{0} \mathcal{E}(-b)$ gives rise to a morphism $\mathcal{O}_{X_{r}} \rightarrow \mathcal{E}(-b)$ and hence if $\mathcal{E}$ is stable, then $0<c_{1}(\mathcal{E}(-b)) / k=\left(c_{1}(\mathcal{E})-k b\right) / k$ and hence $k b-c_{1}<0$. In particular if $\mathcal{E}$ is normalized and stable, then $c_{1}>0$. When $k=2$ the condition $k b(\mathcal{E})-c_{1}(\mathcal{E}) \leq 0$ is in fact equivalent to the semistablity of $\mathcal{E}$. If $k \geq 3$ this is not an equivalence any more.

We can use condition (ii) in Definition 1.2 to translate properties of vector bundles satisfying $\star$ to properties of curves in $X_{r}$. Indeed, for a vector bundle $\mathcal{E}$ satisfying condition $\star$, the choice of $k-1$ global sections whose dependency locus is a curve $C \subset X_{r}$ yields the fundamental exact sequence:

$$
0 \rightarrow \mathcal{O}_{X_{r}}^{k-1} \rightarrow \mathcal{E} \rightarrow \mathcal{J}_{C}\left(c_{1}\right) \rightarrow 0
$$

where $\mathcal{J}_{C}$ is the ideal sheaf of $C \subset X_{r}$. When dualizing (2.4), we get the exact sequence

$$
0 \rightarrow \mathcal{O}_{X_{r}}\left(-c_{1}\right) \rightarrow \mathcal{E}^{\vee} \rightarrow \mathcal{O}_{X_{r}}^{k-1} \rightarrow \omega_{C}\left(5-r-c_{1}\right) \rightarrow 0
$$


where $\omega_{C} \cong \mathcal{E} x t^{2}\left(\mathcal{O}_{C}, \omega_{X_{r}}\right)$ is the dualizing sheaf of $C$. This implies, in particular, that $\omega_{C}\left(5-r-c_{1}\right)$ is generated by $k-1$ global sections. Next we recall the generalized Hartshorne-Serre correspondence (see [3], [23]), which states that is possible to reverse this process, in the sense that one recovers the vector bundle from the surjection in (2.5):

Proposition 2.1. Let $C \subset X_{r}$ be a locally complete intersection curve and assume that, for some $c_{1} \in \mathbb{Z}, \omega_{C}\left(5-r-c_{1}\right)$ has $k-1$ generating global sections. Then there exists a rank $k$ vector bundle $\mathcal{E}$ on $X_{r}$ fitting in the exact sequence (2.4) and the surjection of (2.5) corresponds to the choice of the $k-1$ global sections of $\omega_{C}\left(5-r-c_{1}\right)$. In particular, $c_{1}(\mathcal{E})=c_{1}, c_{2}(\mathcal{E})=\operatorname{deg} C$ and $C$ is the dependency locus of the $k-1$ global sections of $\mathcal{E}$ given by (2.4).

This gives, whenever we have an exact sequence like (2.4), the relation between the two first Chern classes of $\mathcal{E}$ and the degree of $C$. Moreover, the third Chern class of $\mathcal{E}$ is determined by the (arithmetic) genus of the curve $C$ :

Corollary 2.2. If $C \subset X_{r}$ is a locally complete intersection curve associated to a vector bundle on $X_{r}$ of rank $k \geq 2$ as above, then

$$
g(C)=-\frac{5}{2} c_{2}+\frac{1}{2} c_{1} c_{2}+\frac{1}{2} c_{3}+\frac{25}{12} r+\frac{1}{2} r c_{2}-\frac{35}{24} r^{2}+\frac{5}{12} r^{3}-\frac{1}{24} r^{4},
$$

where $c_{i}=c_{i}(\varepsilon), i=1,2,3$. In particular when $r=4$ we have

$$
g(C)=1+\frac{1}{2} c_{1} c_{2}-\frac{1}{2} c_{2}+\frac{1}{2} c_{3} .
$$

Proof. It follows from the equalities $g(C)=1-\chi\left(\mathcal{O}_{C}\right)=1-\chi\left(\mathcal{O}_{X_{r}}\right)+\chi\left(\mathcal{J}_{C}\right)$, $\chi\left(\mathcal{J}_{C}\right)=\chi\left(\mathcal{E}\left(-c_{1}\right)\right)-\chi\left(\mathcal{O}_{X_{r}}^{k-1}\left(-c_{1}\right)\right)$ (by the exact sequence $\left.(2.4)\right)$ and the Riemann-Roch theorem.

Having in mind Hartshorne-Serre correspondence, we will frequently consider pairs

given by a vector bundle $\mathcal{E}$ of rank $k \geq 3$ which satisfies condition $\star$ and a curve $C \subset X_{r}$ dependency locus of $k-1$ global sections of $\mathcal{E}$. We will also say in this case that the pair $(\mathcal{E}, C)$ is a $A C M$ pair and satisfies condition $\star$ (in Proposition 4.1 we will give a criterion for this property in terms of the curve $C)$.

We recall the following generalization of Clifford's theorem (observe that in our situation $C$ is connected because the fact that $\mathcal{E}$ is ACM implies from (2.4) the vanishing of $\left.H^{1} \mathcal{J}_{C}\right)$ :

Theorem 2.3. Let $C$ be a connected locally complete intersection curve, and let $\mathcal{L}$ be a line bundle on $C$ such that $H^{0} \mathcal{L} \neq 0$ and $H^{1} \mathcal{L} \neq 0$. Then $2\left(h^{0} \mathcal{L}-1\right) \leq$ $\operatorname{deg} \mathcal{L}$. 
Proof. The same proof as in [9] Theorem A works in this case, since the irreducibility assumed there is not used to prove the inequality. In fact, we still have a nondegenerate bilinear map $H^{0} \mathcal{L} \otimes \operatorname{Hom}\left(\mathcal{L}, \omega_{C}\right) \rightarrow H^{0} \omega_{C}$, so that the result follows, as in [9], by the so-called bilinear lemma, Serre's duality and Riemann-Roch theorem. The connectedness of $C$ is used to conclude that the dimension $h^{0} \omega_{C}=h^{1} \mathcal{O}_{C}$ is $g(C)$ because $h^{0} \mathcal{O}_{C}=1$.

Let us finish this section by recalling the classification of undecomposable rank two ACM bundles on quartics $X_{4}$ obtained in [15] and on cubics $X_{3}$ obtained in [4].

Proposition 2.4. Let $\mathcal{E}$ be a normalized and undecomposable rank two ACM bundle on a smooth hypersurface $X_{r} \subset \mathbb{P}^{4}$ with Chern classes $c_{i}(\mathcal{E})=c_{i}$, $i=1,2$.

(1) If $r=4$, then $\left(c_{1}, c_{2}\right) \in\{(-1,1),(0,2),(1,3),(1,4),(1,5),(2,8),(3,14)\}$;

(2) if $r=3$, then $\left(c_{1}, c_{2}\right)=\{(0,1),(1,2),(2,5)\}$.

Moreover all the cases arise.

Remark 2.5. In particular in all the above cases condition $\star$ is satisfied with the only exceptions of cases $\left(c_{1}, c_{2}\right) \in\{(-1,1),(0,2),(1,5)\}$ when $r=4$ and $\left(c_{1}, c_{2}\right)=(0,1)$ when $r=3$. Moreover, as shown in [15], it also holds that the vector bundles on $X_{4}$ with $\left(c_{1}, c_{2}\right)=(3,14)$ and the general one with $\left(c_{1}, c_{2}\right)=(2,8)$ are generated by their global sections.

\section{Constructing bundles from extensions}

We prove in this section that direct sums (and more generally extensions) of vector bundles satisfying condition $\star$ are still ACM vector bundles satisfying condition $\star$. We will end by producing in this way examples of rank four ACM vector bundles satisfying condition $\star$ on $X_{4}$. Observe first that an ACM vector bundle $\mathcal{E}$ satisfying condition $\star$ cannot decompose as $\mathcal{E} \cong \mathcal{E}^{\prime} \oplus \mathcal{O}_{X_{r}}(a)$, where $\mathcal{E}^{\prime}$ is a vector bundle of rank $k-1$. Indeed, the possibility $a>0$ is excluded by condition (i), the possibility $a=0$ is excluded by condition (iii) and the possibility $a<0$ is excluded by condition (ii), since the dependency locus of $k-1$ sections of $\mathcal{E}$ would be the dependency locus of $k-1$ sections of $\mathcal{E}^{\prime}$ (hence of expected codimension one).

Of course it can be possible to have direct sums $\mathcal{E} \cong \mathcal{E}^{\prime} \oplus \mathcal{E}^{\prime \prime}$ with $r k\left(\mathcal{E}^{\prime}\right)$ and $r k\left(\mathcal{E}^{\prime \prime}\right) \geq 2$. In fact, we are going to see that the second part in condition (i) implies that condition $\star$ is preserved by direct sums (and more generally by extensions). To show this, we start with a standard fact:

Lemma 3.1. Let $\mathcal{E}^{\prime}$ and $\mathcal{E}^{\prime \prime}$ be two vector bundles on $X_{r}$ of rank $k^{\prime}$ and $k^{\prime \prime}$ respectively. Let $s_{1}^{\prime}, \ldots, s_{k^{\prime}}^{\prime}$ be linearly independent sections of $\mathcal{E}^{\prime}$ and let $s_{1}^{\prime \prime}, \ldots, s_{k^{\prime \prime}}^{\prime \prime}$ be linearly independent sections of $\mathcal{E}^{\prime \prime}$. Assume that the dependency locus of $s_{1}^{\prime}, \ldots, s_{k^{\prime}-1}^{\prime}$ is a curve $C^{\prime}$ and the dependency locus of $s_{2}^{\prime \prime}, \ldots, s_{k^{\prime \prime}}^{\prime \prime}$ is a curve $C^{\prime \prime}$. Then the dependency locus of the sections

$$
\left(s_{1}^{\prime}, 0\right), \ldots,\left(s_{k^{\prime}-1}^{\prime}, 0\right),\left(s_{k^{\prime}}^{\prime}, s_{1}^{\prime \prime}\right),\left(0, s_{2}^{\prime \prime}\right), \ldots,\left(0, s_{k^{\prime \prime}}^{\prime \prime}\right)
$$


of $\mathcal{E}^{\prime} \oplus \mathcal{E}^{\prime \prime}$ is $C^{\prime} \cup C^{\prime \prime} \cup V\left(s_{1}^{\prime} \wedge \cdots \wedge s_{k^{\prime}}^{\prime}, s_{1}^{\prime \prime} \wedge \cdots \wedge s_{k^{\prime \prime}}^{\prime \prime}\right)$, where $s_{1}^{\prime} \wedge \cdots \wedge s_{k^{\prime}}^{\prime}$ is interpreted as a section of the line bundle $\wedge^{k^{\prime}} \mathcal{E}^{\prime}$ and $s_{1}^{\prime \prime} \wedge \cdots \wedge s_{k^{\prime \prime}}^{\prime \prime}$ as a section of $\wedge^{k^{\prime \prime}} \mathcal{E}^{\prime \prime}$.

Proof. It is enough to check the statement locally. We can thus restrict ourselves to an open subset of $X_{r}$ on which the vector bundles trivialize. If on that open set the section $s_{i}^{\prime}$ is represented by the functions $f_{i 1}^{\prime}, \ldots, f_{i k^{\prime}}^{\prime}$ and the section $s_{j}^{\prime \prime}$ is represented by $f_{j 1}^{\prime \prime}, \ldots, f_{j k^{\prime \prime}}^{\prime \prime}$, then the dependency locus of $\left(s_{1}^{\prime}, 0\right), \ldots,\left(s_{k^{\prime}-1}^{\prime}, 0\right),\left(s_{k^{\prime}}^{\prime}, s_{1}^{\prime \prime}\right),\left(0, s_{2}^{\prime \prime}\right), \ldots,\left(0, s_{k^{\prime \prime}}^{\prime \prime}\right)$ is defined by the vanishing of the maximal minors of the $\left(k^{\prime}+k^{\prime \prime}-1\right) \times\left(k^{\prime}+k^{\prime \prime}\right)$ matrix

$$
\left(\begin{array}{cccccc}
f_{11}^{\prime} & \cdots & f_{1 k^{\prime}}^{\prime} & 0 & \cdots & 0 \\
\vdots & & \vdots & \vdots & & \vdots \\
f_{k^{\prime}-1,1}^{\prime} & \cdots & f_{k^{\prime}-1, k^{\prime}}^{\prime} & 0 & \cdots & 0 \\
f_{k^{\prime} 1}^{\prime} & \cdots & f_{k^{\prime} k^{\prime}}^{\prime} & f_{11}^{\prime \prime} & \cdots & f_{1 k^{\prime \prime}}^{\prime \prime} \\
0 & \cdots & 0 & f_{21}^{\prime \prime} & \cdots & f_{2 k^{\prime \prime}}^{\prime \prime} \\
\vdots & & \vdots & \vdots & & \vdots \\
0 & \cdots & 0 & f_{k^{\prime \prime} 1}^{\prime \prime} & \cdots & f_{k^{\prime \prime} k^{\prime \prime}}^{\prime \prime}
\end{array}\right)
$$

These $k^{\prime}+k^{\prime \prime}-1$ minors take the form

$$
\begin{aligned}
& \left|\begin{array}{cccccc}
f_{11}^{\prime} & \ldots & f_{1, i-1}^{\prime} & f_{1, i+1}^{\prime} & \ldots & f_{1 k^{\prime}}^{\prime} \\
\vdots & & \vdots & \vdots & & \vdots \\
f_{k^{\prime}-1,1}^{\prime} & \ldots & f_{k^{\prime}-1, i-1}^{\prime} & f_{k^{\prime}-1, i+1}^{\prime} & \ldots & f_{k^{\prime}-1, k^{\prime}}^{\prime}
\end{array}\right|\left|\begin{array}{ccc}
f_{11}^{\prime \prime} & \ldots & f_{1 k^{\prime \prime}}^{\prime \prime} \\
\vdots & & \vdots \\
f_{k^{\prime \prime} 1}^{\prime \prime} & \ldots & f_{k^{\prime \prime} k^{\prime \prime}}^{\prime \prime}
\end{array}\right|, \quad i=1, \ldots, k^{\prime}, \\
& \left|\begin{array}{ccc}
f_{11}^{\prime} & \ldots & f_{1 k^{\prime}}^{\prime} \\
\vdots & & \vdots \\
f_{k^{\prime} 1}^{\prime} & \ldots & f_{k^{\prime} k^{\prime}}^{\prime}
\end{array}\right|\left|\begin{array}{cccccc}
f_{21}^{\prime \prime} & \ldots & f_{2, j-1}^{\prime \prime} & f_{2, j+1}^{\prime \prime} & \ldots & f_{2 k^{\prime \prime}}^{\prime \prime} \\
\vdots & & \vdots & \vdots & & \vdots \\
f_{k^{\prime \prime} 1}^{\prime \prime} & \ldots & f_{k^{\prime \prime}, j-1}^{\prime \prime} & f_{k^{\prime \prime}, j+1}^{\prime \prime} & \ldots & f_{k^{\prime \prime} k^{\prime \prime}}^{\prime \prime}
\end{array}\right|, \quad j=1, \ldots, k^{\prime \prime} .
\end{aligned}
$$

Since the curve $C^{\prime}$ is locally defined by the minors

$$
\left|\begin{array}{cccccc}
f_{11}^{\prime} & \cdots & f_{1, i-1}^{\prime} & f_{1, i+1}^{\prime} & \ldots & f_{1 k^{\prime}}^{\prime} \\
\vdots & & \vdots & \vdots & & \vdots \\
f_{k^{\prime} 1}^{\prime} & \cdots & f_{k^{\prime}, i-1}^{\prime} & f_{k^{\prime}, i+1}^{\prime} & \cdots & f_{k^{\prime} k^{\prime}}^{\prime}
\end{array}\right|, \quad i=1, \ldots, k^{\prime},
$$

the curve $C^{\prime \prime}$ is defined by the minors

$$
\left|\begin{array}{cccccc}
f_{11}^{\prime \prime} & \cdots & f_{1, j-1}^{\prime \prime} & f_{1, j+1}^{\prime \prime} & \cdots & f_{1 k^{\prime \prime}}^{\prime \prime} \\
\vdots & & \vdots & \vdots & & \vdots \\
f_{k^{\prime} 1}^{\prime \prime} & \cdots & f_{k^{\prime \prime}, j-1}^{\prime \prime} & f_{k^{\prime \prime}, j+1}^{\prime \prime} & \cdots & f_{k^{\prime \prime} k^{\prime \prime}}^{\prime \prime}
\end{array}\right|, \quad j=1, \ldots, k^{\prime \prime}
$$

and the set

is defined by

$$
V\left(s_{1}^{\prime} \wedge \cdots \wedge s_{k^{\prime}}^{\prime}, s_{1}^{\prime \prime} \wedge \cdots \wedge s_{k^{\prime \prime}}^{\prime \prime}\right)
$$

$$
\left|\begin{array}{ccc}
f_{11}^{\prime} & \cdots & f_{1 k^{\prime}}^{\prime} \\
\vdots & & \vdots \\
f_{k^{\prime} 1}^{\prime} & \cdots & f_{k^{\prime} k^{\prime}}^{\prime}
\end{array}\right|
$$


and

the result follows at once.

$$
\left|\begin{array}{ccc}
f_{11}^{\prime \prime} & \cdots & f_{1 k^{\prime \prime}}^{\prime \prime} \\
\vdots & & \vdots \\
f_{k^{\prime \prime} 1}^{\prime \prime} & \cdots & f_{k^{\prime \prime} k^{\prime \prime}}^{\prime \prime}
\end{array}\right|
$$

As a corollary, we can prove the following (see also Lemma 4.1 of [4]):

Lemma 3.2. Let $\mathcal{E}^{\prime}$ and $\mathcal{E}^{\prime \prime}$ be two vector bundles on $X_{r}$ of rank $k^{\prime}$ and $k^{\prime \prime}$ respectively. Assume that $\mathcal{E}^{\prime}$ and $\mathcal{E}^{\prime \prime}$ satisfy condition $\star$ and that there are sections $s_{1}^{\prime}, \ldots, s_{k^{\prime}}^{\prime}$ of $\mathcal{E}^{\prime}$ and $s_{1}^{\prime \prime}, \ldots, s_{k^{\prime \prime}}^{\prime \prime}$ of $\mathcal{E}^{\prime \prime}$ such that the hypersurfaces $V\left(s_{1}^{\prime} \wedge \cdots \wedge s_{k^{\prime}}^{\prime}\right)$ and $V\left(s_{1}^{\prime \prime} \wedge \cdots \wedge s_{k^{\prime \prime}}^{\prime \prime}\right)$ do not share a common component. Then a general $\mathcal{E}$ fitting in an exact sequence

$$
0 \rightarrow \mathcal{E}^{\prime} \rightarrow \mathcal{E} \rightarrow \mathcal{E}^{\prime \prime} \rightarrow 0
$$

satisfies condition $\star$.

Proof. It is clear that $\mathcal{E}$ satisfies conditions (i) and (iii) of Definition 1.2. Hence it is enough to check condition (ii). Since this condition is open, it suffices to prove it for $\mathcal{E}=\mathcal{E}^{\prime} \oplus \mathcal{E}^{\prime \prime}$. But this follows immediately from Lemma 3.1, since our assumption implies that we can find sections $s_{1}^{\prime}, \ldots, s_{k^{\prime}}^{\prime}$ of $\mathcal{E}^{\prime}$ and $s_{1}^{\prime \prime}, \ldots, s_{k^{\prime \prime}}^{\prime \prime}$ of $\mathcal{E}^{\prime \prime}$ such that $V\left(s_{1}^{\prime} \wedge \cdots \wedge s_{k^{\prime}}^{\prime}, s_{1}^{\prime \prime} \wedge \cdots \wedge s_{k^{\prime \prime}}^{\prime \prime}\right)$ is a curve.

Remark 3.3. We can use condition (ii) in Definition 1.2 to give a geometric interpretation (and a criterion) for the hypothesis in Lemma 3.2. For a vector bundle $\mathcal{E}$ satisfying condition $\star$, the choice of $k-1$ global sections $s_{1}, \ldots, s_{k-1}$ whose dependency locus is a curve $C \subset X_{r}$ yields the exact sequence (2.4). Since we are assuming $h^{0} \mathcal{E} \geq k$, this means that there is at least a section $s_{k}$ of $\mathcal{E}$ independent of $s_{1}, \ldots, s_{k-1}$. Any such $s_{k}$ yields a hypersurface of degree $c_{1}$ containing $C$ and defined by the global section $s_{1} \wedge \cdots \wedge s_{k}$ of $\mathcal{O}_{X_{r}}\left(c_{1}\right)$ (with the convention of Lemma 3.1). Notice if $\mathcal{E}^{\prime}$ and $\mathcal{E}^{\prime \prime}$ are ACM also $\mathcal{E}$ is ACM.

Corollary 3.4. Let $\left(\mathcal{E}^{\prime}, C^{\prime}\right)$ and $\left(\mathcal{E}^{\prime \prime}, C^{\prime \prime}\right)$ be two ACM pairs satisfying condition $\star$. Then a general $\mathcal{E}$ appearing in an extension as (3.1) satisfies condition * if at least one of the following conditions is satisfied:

(a) there is a hypersurface of degree $c_{1}^{\prime}$ containing $C^{\prime}$ and a hypersurface of degree $c_{1}^{\prime \prime}$ containing $C^{\prime \prime}$ such that these two hypersurfaces do not share any component;

(b) at least one of $\mathcal{E}^{\prime}$ and $\mathcal{E}^{\prime \prime}$ is generated by its global sections.

Proof. Part (a) is an immediate consequence of Lemma 3.2 and the previous remark. To prove part (b), assume for instance that $\mathcal{E}^{\prime}$ is generated by its global sections. We fix any hypersurface of degree $k^{\prime \prime}$ containing $C^{\prime \prime}$ and take a point in any of its components. Since $\mathcal{E}^{\prime}$ is generated by its global sections we can find $k^{\prime}$ sections of it whose dependency locus does not contain any of those points. This dependency locus is therefore a hypersurface of degree $c_{1}^{\prime}$ 
containing $C^{\prime}$ and having no common components with the fixed hypersurface of degree $c_{1}^{\prime \prime}$ containing $C^{\prime \prime}$. We thus conclude from (a).

We want now to apply Corollary 3.4 to obtain rank four ACM vector bundles on $X_{4}$ satisfying condition $\star$ from vector bundles of rank two $\mathcal{E}^{\prime}$ and $\mathcal{E}^{\prime}$. We recall how to compute the invariants of any $\mathcal{E}$ fitting in an extension (3.1). Let us denote by $c_{i}^{\prime} \in \mathbb{Z}$ the Chern classes of $\mathcal{E}^{\prime}$ and by $c_{i}^{\prime \prime} \in \mathbb{Z}$ the Chern classes of $\mathcal{E}^{\prime \prime}$. Then:

$$
\begin{aligned}
& c_{1}(\mathcal{E})=c_{1}^{\prime}+c_{1}^{\prime \prime}, \\
& c_{2}(\mathcal{E})=c_{2}^{\prime}+4 c_{1}^{\prime} c_{1}^{\prime \prime}+c_{2}^{\prime \prime}, \\
& c_{3}(\mathcal{E})=c_{2}^{\prime} c_{1}^{\prime \prime}+c_{1}^{\prime} c_{2}^{\prime \prime} .
\end{aligned}
$$

Checking Remark 2.5 for finding the list of rank two ACM bundles on $X_{4}$ satisfying condition $\star$ we find the following list of examples.

Example 3.5. We take $\mathcal{E}^{\prime}, \mathcal{E}^{\prime \prime}$ to be rank two ACM vector bundles on $X_{4}$ with $c_{1}=3, c_{2}=14$. These are generated by their global sections, so that it follows from Corollary 3.4(b) that any general element in $\operatorname{Ext}^{1}\left(\mathcal{E}^{\prime \prime}, \mathcal{E}^{\prime}\right)$ (which is a vector space of dimension at least 7) provides a rank four ACM vector bundle satisfying condition $\star$. In fact, in this case it is easier to observe that any extension provides a globally generated vector bundle, and hence it always satisfies condition $\star$. By the formulas (3.2), the invariants of such vector bundle are $\left(k ; c_{1}, c_{2}, c_{3}\right)=(4 ; 6,64,84)$.

Example 3.6. We take now the rank two ACM vector bundles $\mathcal{E}^{\prime}, \mathcal{E}^{\prime \prime}$ in $X_{4}$ with $c_{1}\left(\mathcal{E}^{\prime}\right)=3, c_{2}\left(\mathcal{E}^{\prime}\right)=14$, and $c_{1}\left(\mathcal{E}^{\prime \prime}\right)=2$. It follows again from Corollary $3.4(\mathrm{~b})$ that a general element in $\operatorname{Ext}^{1}\left(\mathcal{E}^{\prime \prime}, \mathcal{E}^{\prime}\right.$ ) (which is a vector space of dimension at least 7 ) provides a rank four ACM vector bundle $\mathcal{E}$ satisfying condition $\star$. In this case, the invariants produced by the formulas (3.2) are $\left(k ; c_{1}, c_{2}, c_{3}\right)=(4 ; 5,46,52)$.

Example 3.7. We repeat the same reasoning as in Example 3.6, but taking now $\mathcal{E}^{\prime \prime}$ a rank two ACM vector bundle with $c_{1}\left(\mathcal{E}^{\prime \prime}\right)=1, c_{2}\left(\mathcal{E}^{\prime \prime}\right)=d$ (for $d=3,4)$. As before, a general element in $\operatorname{Ext}^{1}\left(\mathcal{E}^{\prime \prime}, \mathcal{E}^{\prime}\right)$ (which is a vector space of dimension at least $3 d-8$ ) yields an unstable rank four ACM vector bundle satisfying condition $\star$ and with invariants $\left(k ; c_{1}, c_{2}, c_{3}\right)=(4 ; 4,26+d, 3 d+$ 14), $d=3,4$.

Example 3.8. Now we take $\mathcal{E}^{\prime}, \mathcal{E}^{\prime \prime}$ two normalized rank two ACM vector bundles that are globally generated on $X_{4}$ with $c_{1}=2$ and $c_{2}=8$. Again from Corollary $3.4(\mathrm{~b})$ we get that a general element in $\operatorname{Ext}^{1}\left(\mathcal{E}^{\prime \prime}, \mathcal{E}^{\prime}\right)$ (which is a vector space of dimension at least 4) provides a rank four ACM vector bundle satisfying condition $\star$. Using once more the equations $(3.2)$ we find that this bundle has invariants $\left(k ; c_{1}, c_{2}, c_{3}\right)=(4 ; 4,32,32)$.

Example 3.9. We replace now in Example 3.8 the vector bundle $\mathcal{E}^{\prime \prime}$ with a normalized rank two ACM vector bundle on $X_{4}$ with $c_{1}\left(\mathcal{E}^{\prime \prime}\right)=1$ and $c_{2}\left(\mathcal{E}^{\prime \prime}\right)=d$ 
(with $d=3,4)$. Hence we get that a general element in $\operatorname{Ext}^{1}\left(\mathcal{E}^{\prime \prime}, \mathcal{E}^{\prime}\right)$ (which is a vector space of dimension at least $2 d-4)$ provides an unstable rank four ACM vector bundle satisfying condition $\star$ and with invariants $\left(k ; c_{1}, c_{2}, c_{3}\right)=$ $(4 ; 3,16+d, 8+2 d), d=3,4$.

Example 3.10. Take now $\mathcal{E}^{\prime}$ and $\mathcal{E}^{\prime \prime}$ to be two normalized rank two ACM bundles on $X_{4}$ with $c_{1}\left(\mathcal{E}^{\prime}\right)=1, c_{2}\left(\mathcal{E}^{\prime}\right)=d^{\prime}$ and $c_{1}\left(\mathcal{E}^{\prime \prime}\right)=1, c_{2}\left(\mathcal{E}^{\prime \prime}\right)=d^{\prime \prime}$, (with $d^{\prime}, d^{\prime \prime}=3,4$ ). The curves obtained as the zero loci of sections of $\mathcal{E}^{\prime}$ and $\mathcal{E}^{\prime \prime}$ are elliptic curves of degree $d^{\prime}$ and $d^{\prime \prime}$, in any case contained in at least one hyperplane of $\mathbb{P}^{4}$. A simple calculation shows that a hyperplane section of $X_{4}$ (which is a K3 surface) contains at most a pencil of elliptic curves of degree $d$, while the family of those curves in $X_{4}$ has dimension $d$ (see $[15])$. Hence we can take pairs $\left(\mathcal{E}^{\prime}, C^{\prime}\right)$ and $\left(\mathcal{E}^{\prime \prime}, C^{\prime \prime}\right)$ such that the hyperplanes containing $C^{\prime}$ and $C^{\prime \prime}$ are different. Therefore we can apply Lemma 3.2 and take a general element in $\operatorname{Ext}^{1}\left(\mathcal{E}^{\prime}, \mathcal{E}^{\prime \prime}\right)$ (which has dimension at least $d^{\prime}+d^{\prime \prime}-6 \geq 0$ ) to produce an ACM vector bundle satisfying condition $\star$ and with invariants $\left(k ; c_{1}, c_{2}, c_{3}\right)=\left(4 ; 2, d^{\prime}+d^{\prime \prime}+4, d^{\prime}+d^{\prime \prime}\right), d, d^{\prime}=3,4$.

\section{Constructing bundles from curves on the quartic threefold}

In this section, we use first Hartshorne-Serre correspondence, to translate the property of being an ACM vector bundle satisfying condition $\star$ to the property of curves in $X_{r}$ to be associated to it. This will allow to produce several examples of ACM vector bundles of rank $k=3,4$ on $X_{4}$ satisfying condition $\star$ from curves in $X_{4}$.

We start by characterizing when a curve $C$ determines an ACM vector bundle (observe that the condition $c_{1}>0$ will not be restrictive because of $(5.1)$ ). In the sequel we will denote by $\mathcal{O}_{C}(1)$ the restriction of the hyperplane class $H$ of $X_{r}$ to $C \subset X_{r}$.

Proposition 4.1. Let $C \subset X_{r}$ be a locally complete intersection curve and let $\mathcal{E}$ be the vector bundle of rank $k \geq 2$ obtained, as in Proposition 2.1, from $k-1$ sections of $\omega_{C}\left(5-r-c_{1}\right)$. Assume $c_{1}>0$. Then $\mathcal{E}$ is an ACM bundle satisfying condition $\star$ if and only if $C$ is projectively normal and the following four conditions hold:

(a) the $k-1$ given sections form a basis of $H^{0} \omega_{C}\left(5-r-c_{1}\right)$ (in particular $\left.h^{0} \omega_{C}\left(5-r-c_{1}\right)=k-1\right)$

(b) $h^{0} \mathcal{J}_{C}\left(c_{1}-1\right)=0$ and $h^{0} \mathcal{J}_{C}\left(c_{1}\right) \geq 1$;

(c) $h^{0} \omega_{C}\left(4-r-c_{1}\right)=0$, for which a sufficient condition is $2 g(C)-2<$ $\left(r+c_{1}-4\right) \operatorname{deg} C$

(d) the natural map $\alpha_{n}: H^{0} \omega_{C}\left(5-r-c_{1}\right) \otimes H^{0} \mathcal{O}_{C}(n) \rightarrow H^{0} \omega_{C}\left(5-r-c_{1}+n\right)$ is surjective $\forall n \geq 1$.

Proof. Since $h^{i} \mathcal{O}_{X_{r}}(n)=0$ for all $n \in \mathbb{Z}$ and $i=1,2$ then the condition $h^{1} \mathcal{E}(n)=0$ for all $n \in \mathbb{Z}$ is equivalent to the condition $h^{1} \mathcal{J}_{C}\left(c_{1}+n\right)=0$ for all 
$n \in \mathbb{Z}$, i.e., to the projective normality of $C$. On the other hand, splitting (2.5) into the following exact sequences

$$
0 \rightarrow \mathcal{O}_{X_{r}}\left(-c_{1}\right) \rightarrow \mathcal{E}^{\vee} \rightarrow K \rightarrow 0
$$

and

$$
0 \rightarrow K \rightarrow \mathcal{O}_{X_{r}}^{k-1} \rightarrow \omega_{C}\left(5-r-c_{1}\right) \rightarrow 0
$$

we get that $h^{1} \mathcal{E}^{\vee}(n)=0 \forall n \in \mathbb{Z}$ if and only if the map $r_{n}: H^{0} \mathcal{O}_{X_{r}}^{k-1}(n) \rightarrow$ $H^{0} \omega_{C}\left(5-r-c_{1}+n\right)$ is surjective $\forall n \in \mathbb{Z}$. The surjectivity of $r_{n}$ for all $n<0$ is equivalent to $h^{0} \omega_{C}\left(4-r-c_{1}\right)=0$, for which a sufficient condition is $2 g(C)-2<\left(r+c_{1}-4\right) \operatorname{deg} C$. For $n=0$, the map $r_{0}$ is the one coming from the choice of $k-1$ sections of $\omega_{C}\left(5-r-c_{1}+n\right)$, so it is surjective if and only if we take a system of generators of $H^{0} \omega_{C}\left(5-r-c_{1}+n\right)$. For $n>0$, observe that $r_{n}$ factors through

$H^{0} \mathcal{O}_{X_{r}}^{k-1} \otimes H^{0} \mathcal{O}_{X_{r}}(n) \rightarrow H^{0} \omega_{C}\left(5-r-c_{1}\right) \otimes H^{0} \mathcal{O}_{C}(n) \rightarrow H^{0} \omega_{C}\left(5-r-c_{1}+n\right)$.

If $C$ is projectively normal and $r_{0}$ is surjective, the first map is surjective, and hence the surjectivity of $r_{n}$ becomes equivalent to the surjectivity of the second map, which is precisely $\alpha_{n}$.

Finally, observe that the map $r_{0}$ is not injective (i.e., condition (a) does not hold) is equivalent, by (4.1) and (4.2) and the fact that $c_{1}>0$, to the existence of a section of $\mathcal{E}^{\vee}$ mapping to a nonzero section of $\mathcal{O}_{X_{r}}^{k-1}$. This is equivalent to say that $\mathcal{E}$ has a trivial summand, which means that condition (iii) in Definition 1.2 dos not hold. On the other hand, condition (b) is clearly equivalent, by $(2.4)$, to $h^{0} \mathcal{E}(-1)=0$ and $h^{0} \mathcal{E} \geq k$, i.e., condition (i) in Definition 1.2.

We give now some examples of the above construction applied to $X=X_{4}$ (for the rest of this section, unless otherwise specified, $X$ will stand for $X_{4}$ ). We start by giving a method to construct curves contained in a hyperplane, which we will thus allow us to construct vector bundles with $c_{1}=1$.

Lemma 4.2. Let $\mathcal{C}$ be a family of degenerate curves in $\mathbb{P}^{4}$ such that some curve of $\mathcal{C}$ is contained in a smooth degenerate $\left(\right.$ in $\left.\mathbb{P}^{4}\right)$ quartic surface and it is not the complete intersection of this surface and a hypersurface. Then the general quartic hypersurface $X \subset \mathbb{P}^{4}$ contains a curve of $\mathrm{C}$.

Proof. Consider the set $\Sigma$ of degenerate quartic surfaces of $\mathbb{P}^{4}$ containing a curve of $\mathcal{C}$. We define the natural map

$$
\varphi: \Sigma \rightarrow\left(\mathbb{P}^{4}\right)^{*}
$$

associating to each quartic surface the unique hyperplane containing it. Fix now a hyperplane $H$ of $\mathbb{P}^{4}$. It is a standard fact in the Noether-Lefschetz theory (see for example [6]), that the set of quartic surfaces in $H$ containing a curve in $\mathcal{C}$ has codimension one in the set of quartic surfaces of $H$. In other words, the set $\varphi^{-1}(H)$ has dimension 33, and hence $\Sigma$ has dimension 37 . 
Consider now the set $I$ of pairs $(S, X)$, where $S \in \Sigma$ and $X$ is a quartic hypersurface of $\mathbb{P}^{4}$ containing $S$. Since the fibers of the projection $I \rightarrow \Sigma$ are projective spaces of dimension 35 , it follows that $I$ has dimension 72 .

We finally consider the second projection $p_{2}: I \rightarrow \mathbb{P}^{69}$ (where we identify $\mathbb{P}^{69}$ with the set of quartic hypersurfaces of $\mathbb{P}^{4}$ ). The lemma will be proved if we show that $p_{2}$ is surjective. Observe that, since any smooth degenerate quartic surface in $\mathbb{P}^{4}$ is the hyperplane section of some smooth quartic hypersurface of $\mathbb{P}^{4}$, it follows from our hypotheses that there is a smooth quartic hypersurface $X$ in the image of $p_{2}$. Since a general hyperplane section of $X$ has its Picard group generated by the hyperplane divisor (see for example [19]), such a hyperplane section cannot contain a curve of $\mathcal{C}$, and hence the set $p_{2}^{-1}(X)$ has dimension at most three. Hence the fiber of any element of the image of $p_{2}$ has necessarily dimension three, which shows that $p_{2}$ is surjective, as wanted.

In order to apply the previous lemma we show the following:

Lemma 4.3. A smooth projectively normal space curve $C$ of degree $d$ and genus $g$ with $d \geq g-1$ is contained in a smooth quartic surface in $\mathbb{P}^{3}$.

Proof. The statement follows readily from the more general results of [18]. We give however a direct proof valid for our cases using the following simple standard argument. We first observe, from Castelnuovo-Mumford's criterion, that $J_{C}(4)$ is globally generated. Indeed the projective normality of $C$ provides the vanishing of $h^{1} \mathcal{J}_{C}(3)$, while the vanishings of $h^{3} \mathcal{J}_{C}(1)$ and $h^{2} \mathcal{J}_{C}(2)$ come from the equalities

$$
\begin{gathered}
h^{3} \mathcal{J}_{C}(3)=h^{2} \mathcal{O}_{C}(3)=0, \\
h^{2} \mathcal{J}_{C}(2)=h^{1} \mathcal{O}_{C}(2)=h^{0} \omega_{C}(-2)=0
\end{gathered}
$$

(the latter coming from the assumption $d \geq g-1$ ).

Hence the linear system $\left|H^{0} \mathcal{J}_{C}(4)\right|$ has no base-points outside $C$ and therefore, by Bertini's theorem, a general element of it is smooth outside $C$. By a well known argument (see e.g. [20]), from the exact sequence

$$
0 \rightarrow \mathrm{J}_{C}^{2}(4) \rightarrow \mathrm{J}_{C}(4) \rightarrow N_{C}^{\vee}(4) \rightarrow 0
$$

a surface in the linear system $\left|H^{0} \mathcal{J}_{C}(4)\right|$ provides a section of $N_{C}^{\vee}(4)$, and the singular points of the surface belonging to $C$ are those in the zero locus of the section. The sequence (4.3) proves that the rank two vector bundle $N_{C}^{\vee}(4)$ is generated by the global sections coming from sections of $\mathcal{J}_{C}(4)$. Hence, a general such section of $N_{C}^{\vee}(4)$ will be nowhere vanishing, which implies that a general element of $\left|H^{0} \mathcal{J}_{C}(4)\right|$ is smooth also in the points of $C$. Such a general element gives thus the wanted smooth quartic surface containing $C$.

As a first application, we give a negative answer to Question 1.3.

Proposition 4.4. There exists an ACM bundle on $X$ satisfying condition * with invariants $(4 ; 1,6,4)$. Moreover a general such bundle is not extension class of any rank two ACM bundles. 
Proof. Let $C$ be a smooth projectively normal curve (hence non hyperelliptic) of degree $\operatorname{deg} C=6$ and genus $g(C)=3$ in $\mathbb{P}^{3}$. By Lemma 4.3, $C$ is contained in a smooth quartic surface and by Lemma 4.2 a general quartic hypersurface in $\mathbb{P}^{4}$ contains a degenerate curve $C$ of degree 6 and genus 3 . Since $h^{0} \omega_{C}=3$, by Proposition 2.1 $C$ defines a rank four vector bundle given by

$$
0 \rightarrow \mathcal{O}_{X}^{3} \rightarrow \mathcal{E} \rightarrow \mathcal{J}_{C}(1) \rightarrow 0
$$

with $c_{1}(\mathcal{E})=1, c_{2}(\mathcal{E})=6, c_{3}=4$ (by $(2.6)$ ) and $h^{0} \mathcal{E}=4$. Proposition 4.1 applies once we show the map

$$
\alpha_{1}: H^{0} \omega_{C} \otimes H^{0} \mathcal{O}_{C}(1) \rightarrow H^{0} \omega_{C}(1)
$$

is surjective, which follows by Castelnuovo's Lemma (see [1] p. 151 or [2] theorem (1.6)). Hence $\mathcal{E}$ is ACM and satisfies condition $\star$.

Finally any such bundle is not an extension class of any two rank two ACM bundles on $X$. Suppose to the contrary that there exists a non trivial extension class

$$
0 \rightarrow \mathcal{E}^{\prime} \rightarrow \mathcal{E} \rightarrow \mathcal{E}^{\prime \prime} \rightarrow 0
$$

with $\varepsilon^{\prime}$ and $\varepsilon^{\prime \prime} \mathrm{ACM}$ of rank two. Since $\mathcal{\varepsilon}$ satisfies condition $\star$, then both $\mathcal{E}^{\prime}$ and $\mathcal{E}^{\prime \prime}$ are normalized, i.e., $b\left(\mathcal{E}^{\prime}\right)=b\left(\mathcal{E}^{\prime \prime}\right)=0$ and $h^{0} \mathcal{E}^{\prime} \cdot h^{0} \mathcal{E}^{\prime \prime} \geq 1$. Then a direct computation shows that equations (3.2) have not integral solutions for Chern classes of $\mathcal{E}^{\prime}$ and $\mathcal{E}^{\prime \prime}$ as in Proposition 2.4 and we are done.

Remark 4.5. Alternatively, to show the existence of space curves of degree 6 and genus 3 on general $X$ one can start with a curve of degree 10 as in Example 3.10 and then taking the residual curve to it in a complete intersection $(2,2,4)$.

Similar to the previous case, we have also the following:

Proposition 4.6. There exists an ACM bundle on $X$ satisfying condition $\star$ with invariants $(3 ; 1,5,2)$.

Proof. Let $C$ be a smooth curve of type $(2,3)$ in a smooth quadric surface $C \subset Q \subset \mathbb{P}^{3}$. By Lemma 4.3, there exists a smooth quartic surface containing $C$. Hence, by Lemma 4.2, a general quartic hypersurface contains a curve $C$ of degree 5 genus 2. Since $h^{0} \omega_{C}=2$, by Proposition 2.1 we get a rank three vector bundle $\mathcal{E}$ fitting in the exact sequence

$$
0 \rightarrow \mathcal{O}_{X}^{2} \rightarrow \mathcal{E} \rightarrow \mathcal{J}_{C}(1) \rightarrow 0
$$

with $c_{1}=1, c_{2}=5, c_{3}=2$ and $h^{0} \mathcal{E}=3$. Using Castelnuovo's Lemma it is easy to check that $C$ satisfies the conditions of Proposition 4.1 and hence $\mathcal{E}$ is $\mathrm{ACM}$ and satisfies condition $\star$.

Remark 4.7. A similar liaison argument as above shows the existence of rational space cubic curves in $X$. Specifically, let $Q$ be the unique quadric surface containing a quintic $C \subset X$ of genus 2 as in Proposition 4.6. Then $C$ is residual to a rational cubic curve $D \subset Q \cap X$. 


\section{Bounds of the Chern classes}

In this section we will prove several restrictions for the Chern classes of ACM vector bundles, with special attention to the case $r=4$. This will yield the list of possible Chern classes stated in Theorem 1.5 for the cases $k=3$, 4 . We start with bounds for $c_{1}$ and $c_{2}$. The proof of the bound for $c_{1}$ is a straightforward extension of the method used in [14] for the rank two case.

Lemma 5.1. Let $(\mathcal{E}, C)$ be an $A C M$ pair on $X_{r}$ satisfying condition $\star$ and having invariants $\left(k ; c_{1}, c_{2}, c_{3}\right)$. Then

$$
1 \leq c_{1} \leq \frac{k(r-1)}{2}
$$

and

$$
c_{2} \leq \frac{r}{2} c_{1}^{2}-\frac{r(r-2)}{2} c_{1}+\frac{r(r-1)(r-2)}{6} k .
$$

Proof. By the exact sequence (2.4), since $h^{0} \mathcal{E} \geq k$ it follows $h^{0} \mathcal{J}_{C}\left(c_{1}\right) \geq 1$ and hence $c_{1} \geq 1$. Let $H$ be a general hyperplane section of $X_{r}$. Taking cohomology in the exact sequence

$$
0 \rightarrow \mathcal{E}(-2) \rightarrow \mathcal{E}(-1) \rightarrow \mathcal{E}_{H}(-1) \rightarrow 0
$$

(and its twists by any $\mathcal{O}_{X_{r}}(l)$ ), we get that $\mathcal{E}_{H}$ is an ACM bundle on $H$. Since $h^{0} \mathcal{E}(-1)$ (because $\mathcal{E}$ normalized) we also get $h^{0} \mathcal{E}_{H}(-1)=0$. Therefore,

$$
0 \leq h^{2} \mathcal{E}_{H}(-1)=\chi \mathcal{E}_{H}(-1)=-c_{2}+\frac{r}{2} c_{1}^{2}-\frac{r(r-2)}{2} c_{1}+\frac{r(r-1)(r-2)}{6} k
$$

and the bound for $c_{2}$ follows. Similarly, if $Y$ is a general hyperplane section of $H$, we have an exact sequence

$$
0 \rightarrow \mathcal{E}_{H}(-2) \rightarrow \mathcal{E}_{H}(-1) \rightarrow \mathcal{E}_{Y}(-1) \rightarrow 0
$$

which implies $h^{0} \mathcal{E}_{Y}(-1)=0$. Therefore $0 \geq \chi \mathcal{E}_{Y}(-1)=r\left(c_{1}-k(r-1) / 2\right)$ and the upper bound for $c_{1}$ follows immediately.

In the case of quartic hypersurfaces $X_{4}$, which is the case we are interested in, we have stronger restrictions:

Proposition 5.2. Let $(\mathcal{E}, C)$ be an $A C M$ pair satisfying condition $\star$ on $X_{4}$ and having invariants $\left(k ; c_{1}, c_{2}, c_{3}\right)$. Then

$$
c_{3}=-\frac{4}{3} c_{1}^{3}+2 c_{1}^{2}-\frac{14}{3} c_{1}+c_{1} c_{2}-c_{2}+2 k
$$

and

$$
g(C)=-\frac{2}{3} c_{1}^{3}+c_{1}^{2}-\frac{7}{3} c_{1}+1+\left(c_{1}-1\right) c_{2}+k .
$$

Moreover

$$
1 \leq c_{1} \leq \frac{3 k}{2}
$$




$$
2 c_{1}^{2}-2 c_{1}+k \leq c_{2} \leq \min \left\{2 c_{1}^{2}-4 c_{1}+4 k, 2 c_{1}^{2}+k\right\}
$$

and if $c_{1}>1$, then $c_{2} \geq 2 c_{1}^{2}-4 c_{1}+8$.

Proof. By hypothesis $h^{0} \mathcal{E}(-1)=0$, and also $h^{0} \mathcal{E}^{\vee}=0$ because $\mathcal{E}$ has not trivial summands (see the proof of Proposition 4.1). Since $h^{3} \mathcal{E}(-1)=h^{0} \mathcal{E}^{\vee}$ (this is the point in which we use $r=4$ ) it follows $\chi \mathcal{E}(-1)=0$, which provides (5.2) by using Riemann-Roch. Now (5.3) comes from (2.6) performing the substitution of $c_{3}$ given by (5.2).

The bound (5.4) and first upper bound for $c_{2}$ in (5.5) are the ones of Lemma 5.1 for $r=4$. For the other upper bound for $c_{2}$, since $h^{3} \mathcal{E}=h^{0} \mathcal{E}^{\vee}(-1)$ $=0$ (again because $r=4$ ) and $h^{0} \mathcal{E} \geq k$ by hypothesis, if follows that $\chi(\mathcal{E}) \geq k$. By Riemann-Roch, using the substitution (5.2) we get $\chi(\mathcal{E})=-c_{2}+2 c_{1}^{2}+2 k$ which yields the wanted upper bound for $c_{2}$.

For the lower bound of $c_{2}$, we take a general linear projection $\pi: X \rightarrow \mathbb{P}^{3}$ from a point of $\mathbb{P}^{4}$. It is immediate to observe that $\pi_{*} \mathcal{E}$ is an ACM vector bundle of rank $4 k$, which should split completely by Horrocks theorem. Since $h^{0} \mathcal{E}(-1)=0, \pi_{*} \mathcal{E}$ must contain a direct summand $\mathcal{O}_{X_{4}}^{h^{0} \mathcal{E}}$, and similarly a direct summand $\mathcal{O}_{X_{4}}(-2)^{h^{3} \mathcal{E}(-2)}$, since $h^{3} \mathcal{E}(-1)=0$. Therefore, $h^{0} \mathcal{E}+h^{3} \mathcal{E}(-2) \leq 4 k$, which gives the wanted inequality when using Riemann-Roch and the substitution (5.2).

Finally, let us prove the last lower bound for $c_{2}$. Since $h^{0} \mathcal{J}_{C}\left(c_{1}-2\right)=0$ (by Proposition 4.1(b)), it follows that

$$
h^{0} \mathcal{O}_{C}\left(c_{1}-2\right)=h^{0} \mathcal{O}_{X_{4}}\left(c_{1}-2\right)=\frac{2}{3} c_{1}^{3}-3 c_{1}^{2}+\frac{19}{3} c_{1}-5 .
$$

Since by Riemann-Roch we have

$$
\chi \mathcal{O}_{C}\left(c_{1}-2\right)=\frac{2}{3} c_{1}^{3}-c_{1}^{2}+\frac{7}{3} c_{1}-k-c_{2}
$$

we derive $h^{0} \omega_{C}\left(2-c_{1}\right)=c_{2}-2 c_{1}^{2}+4 c_{1}-5+k$. The proof concludes by applying the bilinear lemma (see [9]) to the nondegenerate bilinear map

$$
H^{0} \omega_{C}\left(1-c_{1}\right) \otimes H^{0} \mathcal{O}_{C}(1) \rightarrow H^{0} \omega_{C}\left(2-c_{1}\right)
$$

using that $h^{0} \omega_{C}\left(1-c_{1}\right)=k-1$ (by Proposition 4.1(a)) and that $h^{0} \mathcal{O}_{C}(1) \geq 5$ if $c_{1}>1$, because $C$ is not contained in any hyperplane (by Proposition 4.1(b)).

We will study separately the cases $c_{1}=1$ and $2 \leq c_{1} \leq 3 k / 2$. Proposition 5.2 gives immediate results for vector bundles of rank $k \geq 3$ with $c_{1}=1$ :

Proposition 5.3. Let $(\mathcal{E}, C)$ be a pair on $X_{4}$ satisfying condition $\star$, where $\mathcal{E}$ is $\mathrm{ACM}$ of rank $k \geq 3$ and $c_{1}=1$. Then $c_{2}=k+2, c_{3}=2 k-4$ and $C$ is a curve of degree $k+2$ and genus $g(C)=k-1$.

Proof. We immediately get from Proposition $5.2 g(C)=k-1$ and $\operatorname{deg} C=$ $c_{2} \leq k+2$. Hence, $C$ cannot be a plane curve and therefore $h^{0} \mathcal{J}_{C}(1)=1$ (it is at least 1 by Proposition 4.1). This implies $\left(h^{3} \mathcal{E}=0\right.$, as proved in 
Proposition 5.2) $\chi(\mathcal{E})=h^{0} \mathcal{E}=k-1+h^{0} \mathcal{J}_{C}(1)=k$. Since by Riemann-Roch $\chi(\mathcal{E})=-c_{2}+2 k+2$ it follows $c_{2}=k+2$, which is the degree of $C$. Finally, from (5.2) we get $c_{3}=2 k-4$.

For the rank three case when $c_{1}>1$ we have the following result if $c_{1}=3,4$ :

Proposition 5.4. Let $(\mathcal{E}, C)$ be a pair satisfying condition $\star$, where $\mathcal{E}$ is ACM of rank $k=3$. If $c_{1} \geq 3$, then $2 c_{1}^{2}-4 c_{1}+11 \leq c_{2} \leq 2 c_{1}^{2}-4 c_{1}+12$.

Proof. The upper bound follows from Proposition 5.2. For the lower bound, observe that $h^{3} \mathcal{E}^{\vee}(1)=h^{0} \mathcal{E}(-2)=0$, and thus $h^{0} \mathcal{E}^{\vee}(1)=\chi \mathcal{E}^{\vee}(1)=2 c_{1}^{2}-$ $4 c_{1}-c_{2}+12$. Hence it will be enough to prove $h^{0} \mathcal{E}^{\vee}(1) \leq 1$.

Assume for contradiction $h^{0} \mathcal{E}^{\vee}(1) \geq 2$ and let $s_{1}, s_{2}$ be two independent global sections. Then $\wedge^{2}\left(\mathcal{E}^{\vee}(1)\right) \cong \mathcal{E}\left(2-c_{1}\right)$ contains the nonzero section defined by $s_{1} \wedge s_{2}$, which is absurd since $\mathcal{E}$ is normalized and $2-c_{1}<0$.

Remark 5.5. The above proposition leaves only two possibilities for $c_{2}$, depending on whether $h^{0} \mathcal{E}^{\vee}(1)$ is 0 or 1 . When $h^{0} \mathcal{E}^{\vee}(1)=0$ (i.e., $c_{2}=2 c_{1}^{2}-4 c_{1}+12$ ), it follows that both $\mathcal{E}(-1)$ and its dual have no global sections, so it follows (as in [21] Remark 1.2.6) that $\mathcal{E}(-1)$, and hence $\mathcal{E}$, is stable. Observe that if $c_{1}=3$, we get $c_{2}=18$ and if $c_{1}=4$, then $c_{2}=28$.

Propositions 5.2, 5.3, and 5.4 give immediately the following possibilities for the Chern classes of ACM vector bundles of rank three or four over $X_{4}$ :

Proposition 5.6. Let $(\mathcal{E}, C)$ be an $\mathrm{ACM}$ pair satisfying condition $\star$ where $\mathcal{E}$ is of rank $k=3$. Then the possibilities for the Chern classes of $\mathcal{E}$ and the genus $g=g(C)$ are:
(1) $c_{1}=1, c_{2}=5, c_{3}=2, g(C)=2$;
(2) $c_{1}=2,8 \leq c_{2} \leq 11, c_{3}=c_{2}-6, g(C)=c_{2}-2$;
(3) $c_{1}=3,17 \leq c_{2} \leq 18, c_{3}=2 c_{2}-26, g(C)=2 c_{2}-12$;
(4) $c_{1}=4,27 \leq c_{2} \leq 28, c_{3}=3 c_{2}-66, g(C)=3 c_{2}-32$.

Proposition 5.7. Let $(\mathcal{E}, C)$ be an $\mathrm{ACM}$ pair satisfying condition $\star$ where $\mathcal{E}$ is of rank $k=4$. Then the possibilities for the Chern classes of $\mathcal{E}$ and the genus $g=g(C)$ are:
(1) $c_{1}=1, c_{2}=6, c_{3}=4, g(C)=3$;
(2) $c_{1}=2,8 \leq c_{2} \leq 12, c_{3}=c_{2}-4, g(C)=c_{2}-1$;
(3) $c_{1}=3,16 \leq c_{2} \leq 22, c_{3}=2 c_{2}-24, g(C)=2 c_{2}-11$;
(4) $c_{1}=4,28 \leq c_{2} \leq 32, c_{3}=3 c_{2}-64, g(C)=3 c_{2}-31$
(5) $c_{1}=5,44 \leq c_{2} \leq 46, c_{3}=4 c_{2}-132, g(C)=4 c_{2}-65$
(6) $c_{1}=6, c_{2}=64, c_{3}=84, g(C)=5 c_{2}-117$.

We end this section with some remarks and comments. We notice the following result can be derived using similar arguments as in the above cases of rank three: 
Corollary 5.8. There are no rank three ACM vector bundles satisfying condition $\star$ on a general quartic hypersurface in $\mathbb{P}^{n}, n \geq 5$. In other words, rank three ACM bundles satisfying condition $\star$ on a general quartic threefold $X_{4} \subset \mathbb{P}^{4}$ do not extend to a general quartic hypersurface $W \subset \mathbb{P}^{5}$ having $X_{4}$ as a hyperplane section.

Since in [8] we showed that all rank two ACM bundles on a general quartic $W \subset \mathbb{P}^{n}, n \geq 5$, splits, then a natural question, which we hope to consider later, is the following:

Question 5.9. Which is the minimum rank for a positive dimensional family of ACM bundles satisfying condition $\star$ on a general quartic fourfold $W \subset \mathbb{P}^{5}$ ?

\section{References}

[1] E. Arbarello, M. Cornalba, P. A. Griffiths, and J. Harris, Geometry of Algebraic Curves. Vol. I, Springer-Verlag, New York, 1985.

[2] E. Arbarello and E. Sernesi, Petri's approach to the study of the ideal associated to a special divisor, Invent. Math. 49 (1978), no. 2, 99-119.

[3] E. Arrondo, A home-made Hartshorne-Serre correspondence, Rev. Mat. Complut. 20 (2007), no. 2, 423-443.

[4] E. Arrondo and L. Costa, Vector bundles on Fano 3-folds without intermediate cohomology, Comm. Algebra 28 (2000), no. 8, 3899-3911.

[5] E. Arrondo and D. Faenzi, Vector bundles with no intermediate cohomology on Fano threefolds of type $V_{22}$, Pacific J. Math. 225 (2006), no. 2, 201-220.

[6] J. Carlson, M. Green, P. Griffiths, and J. Harris, Infinitesimal variations of Hodge structure. I, Compositio Math. 50 (1983), no. 2-3, 109-205.

[7] L. Chiantini and C. Madonna, A splitting criterion for rank 2 bundles on a general sextic threefold, Internat. J. Math. 15 (2004), no. 4, 341-359.

$[8] \_$, ACM bundles on general hypersurfaces in $\mathbb{P}^{5}$ of low degree, Collect. Math. 56 (2005), no. 1, 85-96.

[9] D. Eisenbud, J. Koh, and M. Stillman, Determinantal equations for curves of high degree, Amer. J. Math. 110 (1988), no. 3, 513-539.

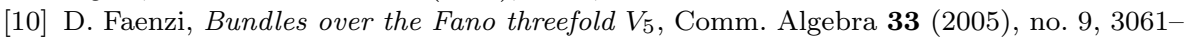
3080.

[11] G. Horrocks, Vector bundles on the punctured spectrum of a local ring, Proc. London Math. Soc. (3) 14 (1964), 689-713.

[12] A. Iliev and D. Markushevich, Quartic 3-fold: Pfaffians, vector bundles, and halfcanonical curves, Michigan Math. J. 47 (2000), no. 2, 385-394.

[13] N. M. Kumar, A. P. Rao, and G. V. Ravindra, Arithmetically Cohen-Macaulay bundles on three dimensional hypersurfaces, Int. Math. Res. Not. IMRN 2007, no. 8, Art. ID rnm025, $11 \mathrm{pp}$.

[14] C. Madonna, A splitting criterion for rank 2 vector bundles on hypersurfaces in $P^{4}$, Rend. Sem. Mat. Univ. Politec. Torino 56 (1998), no. 2, 43-54 (2000).

[15] _ Rank-two vector bundles on general quartic hypersurfaces in $\mathbb{P}^{4}$, Rev. Mat. Complut. 13 (2000), no. 2, 287-301.

[16] _ ACM vector bundles on prime Fano threefolds and complete intersection Calabi-Yau threefolds, Rev. Roumaine Math. Pures Appl. 47 (2002), no. 2, 211-222.

[17] _ Rank 4 vector bundles on the quintic threefold, Cent. Eur. J. Math. 3 (2005), no. $3,404-411$.

[18] S. Mori, On degrees and genera of curves on smooth quartic surfaces in $P^{3}$, Nagoya Math. J. 96 (1984), 127-132. 
[19] B. G. Moǔšezon, Algebraic homology classes on algebraic varieties, Izv. Akad. Nauk SSSR Ser. Mat. 31 (1967), 225-268.

[20] P. E. Newstead, A space curve whose normal bundle is stable, J. London Math. Soc. (2) 28 (1983), no. 3, 428-434.

[21] C. Okonek, M. Schneider, and H. Splinder, Vector Bundles on Complex Projective Spaces, Progress in Mathematics, 3. Birkhauser, Boston, Mass., 1980.

[22] G. Ottaviani, Some extensions of Horrocks criterion to vector bundles on Grassmannians and quadrics, Ann. Mat. Pura Appl. (4) 155 (1989), 317-341.

[23] A. Vogeelar, Constructing vector bundles from codimension-two subvarieties, $\mathrm{PhD}$ thesis, Leiden, 1978.

ENRIQUE ARRONDO

Departamento de Álgebra

Facultad de Ciencias Matemáticas

Universidad Complutense de Madrid

28040 MADRID, SPAIN

E-mail address: Enrique_Arrondo@mat.ucm.es

Carlo G. Madonna

ICMAT, CSIC-UAM-UC3M-UCM

C/ Serrano 121

28006 Madrid, Spain

CURRENT ADDRESS:

Departamento de Álgebra

Facultad de Ciencias Matemáticas

Universidad COMPLUtense de Madrid

28040 Madrid, SPAIN

E-mail address: carlo.madonna@mat.csic.es 